\title{
Expression of angiogenesis-stimulating factors (VEGF, CD31, CD105) and angiogenetic index in gingivae of patients with chronic periodontitis
}

\author{
Aldona Kasprzak¹, Anna Surdacka², Maciej Tomczak², Wieslawa Przybyszewska1, \\ Agnieszka Seraszek-Jaros ${ }^{3}$, Agata Malkowska-Lanzafame², \\ Elzbieta Siodla ${ }^{1}$, Elzbieta Kaczmarek ${ }^{3}$
}

${ }^{1}$ Chair and Department of Histology and Embryology, Poznan University of Medical Sciences in Poznan, Poland

${ }^{2}$ Chair and Department of Conservative Dentistry and Periodontology, Poznan University of Medical Sciences in Poznan, Poland

${ }^{3}$ Department of Bioinformatics and Computational Biology, Chair of Clinical Pathomorphology, Poznan University of Medical Sciences in Poznan, Poland

\begin{abstract}
The aim of this study was to determine the prognostic value of the angiogenesis rate in chronic periodontitis (CP). A total of 61 human gingival samples were taken from patients with $\mathrm{CP}(\mathrm{n}=40)$ obtained during open curettage with gingivectomy, healthy periodontia $(n=15)$, and reactive lymph nodes $(n=7)$. Quantitative immunocytochemistry studies of VEGF, CD31 (PECAM-1) and CD105 (endoglin) were performed using the spatial visualization method. CD105/CD31 and VEGF/CD31 angiogenetic ratios (ARs) were established to determine the proliferation fraction of the endothelium. In patients with $\mathrm{CP}$, the proliferation of blood vessels was observed, including the presence of numerous high endothelial venules (HEVs) and ordinary vessels. In gingival HEVs of patients with CP, the higher expression was shown by CD31 and, in turn CD105 and VEGF. The entire vascular expression of CD31 in the gingiva correlates with grading in lamina propria, but our study failed to document correlations between the expression of VEGF and CD105 and clinical data of patients with $\mathrm{CP}$. Higher ARs were seen in gingivae of CP patients compared to controls. We concluded that overexpression of the angiogenesis-associated factors in $\mathrm{CP}$ suggests its significance in protracting the inflammatory process or periodic exacerbations of the process and destruction of the periodontium. The increased CD105/CD31 and VEGF/CD31 ratios in gingiva confirms an augmented proliferative fraction of the endothelium in gingiva with CP. (Folia Histochemica et Cytobiologica 2012, Vol. 50, No. 4, 554-564)
\end{abstract}

Key words: chronic periodontitis, angiogenetic ratio, HEVs, immunocytochemistry, spatial visualization technique

\section{Introduction}

Bacterial and viral infections represent the main etiological factor in the development of chronic perio-

Correspondence address: A. Kasprzak,

Department of Histology and Embryology,

University of Medical Sciences,

Swiecickiego St. 6, 60-781 Poznan, Poland;

tel.: + 486185464 41, fax: + 486185464 40;

e-mail: akasprza@ump.edu.pl dontitis (CP), but the progress and intensity of the disease are strictly related to the immune reaction of the body to foreign antigens $[1,2]$. Periodontitis induces destruction of periodontal tissue connective matrix, loss of fibrous attachment, alveolar bone resorption and an impaired formation of new bone [3]. Risk factors for moderate to severe periodontitis that have been identified include cigarette smoking, advancing age, diabetes mellitus and certain other systemic conditions [1]. A relationship has been demonstrated between an increasing number of gingival 
blood vessels and the progression of chronic periodontitis $[4,5]$. On the other hand, periodontitis is an independent risk factor for a systemic vascular disease and may reflect stimulation of acute phase protein synthesis by cytokines released by periodontal high endothelial cells (HECs) [4, 6, 7]. Periodontal diseases have many influences on the occurrence and severity of the other systemic disorders, such as respiratory conditions and diabetes mellitus [8].

The quantitative vascular alterations were demonstrated to be accompanied by qualitative changes, involving an appearance of high endothelial post-capillary venules (HEVs, HEV-like venules, periodontal HEVs) $[5,9,10]$. The HEVs were concluded to represent sites of migration and lymphocyte recirculation in the inflamed periodontal tissues. No such blood vessels were noted in healthy gingivae, and their appearance was associated with the progression of tissue inflammation [10]. Blood supply to the gingiva plays a principal role in the degradation of periodontal tissues and fully justifies the categorization of chronic periodontitis as a vasoproliferative disease $[5,11]$.

Vascular endothelial growth factor (VEGF) represents a specific mitogen of endothelial cells in vitro and an inducer of angiogenesis in vivo $[12,13]$. It can also be regarded as a factor of initiation and progression of gingivitis to periodontitis, i.e. through expansion of the blood vessel network [14]. Studies point to a correlation between VEGF concentration in gingival fluid and clinical parameters in CP patients (gingival index, CAL, radiologically detected bone loss) [15]. An expression of VEGF in gingival tissue higher than that in the controls has been observed also in the gingivae of diabetic patients and patients with periodontal diseases [16]. A relationship has been suggested between VEGF and the etiology of early phases of periodontitis [17]. A higher concentration of VEGF has been demonstrated in gingival crevicular fluid compared to serum, which would point to a local production of VEGF [18]. Immunocytochemical techniques permitted the detection of significant differences in localization and distribution of VEGF within gingivae with minimal inflammatory lesions and in advanced periodontitis [19]. VEGF expression was significantly increased in epithelial and endothelial cells from non-diabetic patients as well as diabetic patients with chronic, generalized, severe periodontitis, compared to control samples. A significantly higher number of VEGF-positive cells was observed in patients with type 1 diabetes mellitus and periodontitis versus non-diabetic patients with chronic periodontitis [20]. The data of Artese et al. (2010) suggests that VEGF is an important factor in the pathogenesis of the aggressive and chronic forms of peri- odontitis, but further investigations with a large population sample are needed to identify how VEGF is involved in the tissue inflammation-associated processes [21]. In pathological tissues, various cellular sources of VEGF have been documented [19, 20-22].

The platelet-endothelial cell adhesion molecule1 (PECAM-1, CD31) represents one of the adhesion molecules which manifests the highest expression in endothelial cells (ECs), playing a key role in adhesion between ECs and in interactions of the cells with leukocytes $[23,24]$. The transmigration of lymphocytes through the HEV wall takes place under the effect of PECAM-1 [25]. The available literature contains individual investigations into expressions of PECAM-1 and endoglin (CD105). The molecules served mainly to 'mark' gingival blood vessels $[24,26$, 27]. The expression of PECAM-1 has been demonstrated mainly on lymphocytes and ECs in patients with gingivitis and periodontitis [26].

The functions of endoglin are linked to angiogenesis and ECs phenotype differentiation, adhesion and cell migration as well as to the maintenance of homeostasis of vascular walls [28, 29]. The available literature on the subject lacks studies related to the role of endoglin in periodontal inflammatory processes, although its expression has been described in ECs of pulpar blood vessels in patients with CP [27].

The role of angiogenesis stimulating factors in chronic periodontitis is poorly recognised. Our earlier immunocytochemical studies pointed to their potential role as selective markers of blood vessels (including HEVs) in gingiva in pathogenesis of CP [9].

In this study, we investigated the expression of endothelial cell marker CD31 (PECAM-1) and two angiogenesis-associated molecules: vascular endothelial growth factor (VEGF) and CD105 (endoglin) in the gingivae of patients with chronic periodontitis by immunohistochemistry. The aim of the study was to determine the prognostic value of the angiogenesis rate in chronic periodontitis. Endoglin/CD31 and $\mathrm{VEGF} / \mathrm{CD} 31$ angiogenesis ratios (AR1 and AR2) were established to determine the proliferating fraction of endothelial cells.

\section{Material and methods}

This study was performed on 40 patients (23 women and 17 men, mean age: $43 \pm 11$ years) with chronic periodontitis. In every patient 1-4 fragments of gingiva (a total of 61 gingival fragments) were sampled from the vicinity of various teeth with variable values of clinical attachment level/loss (CAL): CAL I $-\leq 3 \mathrm{~mm}$ (6 gingival fragments), CAL II - 4-6 mm (10 fragments), CAL III $-\geq 7$ mm (45 fragments). Advancement of the disease was determined using 
CAL measurements employing the Florida Probe ${ }^{\circledR}$ instrument. Most of the analyzed fragments originated from patients with $\mathrm{CP}$ with the highest value of clinical attachment/ /loss (CAL III) (74\% gingival fragments). Gingival samples from all patients were isolated using a scalpel from various sextants, from the vicinity of various maxillary and mandibular teeth in the course of open curettage with gingivectomy, following a successful completion of the hygienization phase in the periodontal treatment (API index $<15 \%$ ). The control material (negative control, C1) included gingival samples isolated from 15 patients with clinically healthy periodontia. Tissue material from control patients was obtained in the course of the extraction of teeth affected by retention, during surgical creation of the so-called 'orthodontic window', or in the course of the procedure of clinical crown elongation. Positive control (C2) for HEV type blood vessels involved reactive lymph nodes samples from seven patients. Informed consent was obtained from the patients and the Bioethical Commission of the Medical University approved the procedure of the study.

Tissue material was fixed for 24 hours in $4 \%$ buffered formalin and then embedded in paraffin. Serial $5 \mu \mathrm{m}$ thick sections were deposited onto SuperFrost/Plus microscope glasses. Routine staining of sections using hematoxylin and eosin $(\mathrm{H}+\mathrm{E})$ was performed for morphological evaluation of gingivae and to qualify them for immunocytochemical examination. Particular attention was devoted to postcapillary venules with high endothelium (HEV, HEV-like) and to typical (standard) gingival blood vessels lined with the typical flat endothelium. The size and distribution of inflammatory infiltrate were evaluated. Intensity of inflammatory lesions (grading) was estimated using the four grade semi-quantitative scale, with 3 points given for an intense, including most frequently dispersed, infiltrate, 2 points for a moderately intense but also dispersed infiltrate, 1 point for individual dispersed or focally arranged inflammatory cells in lamina propria, and 0 points for the absence of cells typical of inflammatory infiltrate.

For the detection and examination of cellular localization of all three antigens, the classical ABC technique [(strept)-avidin-biotinylated peroxidase complex] was used, as described in detail in other papers [9, 30, 31]. Immunocytochemical studies (separate for every vascular marker) were performer on 61 serial paraffin sections of gingivae sampled from CP patients (40 patients), 15 gingival samples with normal periodontium (15 patients), and seven sections originating from reactive lymph nodes (seven patients).

The ABC method was conducted using monoclonal mouse antibodies (mAbs) specific for the examined proteins and in optimal dilutions: PECAM-1 (1:50), VEGF (clone VG1, 1:50) (both from DAKO), and endoglin (1:100) (Novocastra Labs). The sections were treated with primary mAbs at night at $4^{\circ} \mathrm{C}$, then with secondary biotinylated link antimouse and anti-rabbit IgG and with the ABC complex (Dako
REAL $^{\mathrm{TM}}{ }^{\text {Envision }}{ }^{\mathrm{TM}}$, Dako, Glostrup, Denmark). Following deparaffination and rehydration, the preparations were additionally boiled in $10 \mathrm{mM}$ citrate buffer in a $700 \mathrm{~W}$ microwave oven for $18 \mathrm{~min}$, washed in PBS and then subjected to the reaction according to the standard procedure. Every test was accompanied by a negative control in which specific antibodies were substituted by a normal serum of a respective species in $0.05 \mathrm{M}$ Tris- $\mathrm{HCl}, \mathrm{pH} \sim 7.6$, supplemented with $0.1 \%$ bovine serum albumin (BSA) and $15 \mathrm{mM}$ sodium azide.

Microscopic image analysis. The preparations were examined under a light microscope Olympus BH-2 with an inbuilt digital camera. On the basis of a result obtained in immunocytochemical reaction, a morphometric analysis included 61 gingival preparations with expression of endoglin (obtained from all 40 patients with $\mathrm{CP}$ ), 26 preparations with expression of PECAM-1 (obtained from 22 of 40 patients), and 42 preparations with expression of VEGF (obtained from 33 of 40 patients). In the control (C1) group with a healthy periodontium, the morphometric analysis was performed in 15 gingival preparations with expression of endoglin, six preparations with expression of PECAM-1, and seven preparations with expression of VEGF. In the C2 group (seven reactive lymph nodes, seven patients) the morphometric analysis included seven preparations with expression of every marker. Color microscope images were recorded and archived at objective magnification of $40 \times$ (at least ten fields of every preparation with immunocytochemical reaction for presence of a given marker (with at least five fields with $\mathrm{HEVs}$ and five fields with ordinary blood vessels) using the method programmed in $\mathrm{C}++$ language by Strzelczyk for spatial marker visualization in microscope images, worked out and implemented in A4D computer software [9, 32]. The morphometric studies included a total of 1,290 microscope images in the CP group, 280 images in the $\mathrm{C} 1$ group, and 210 in the $\mathrm{C} 2$ group. The A4D software allowed the estimation at the end mean surface area of immunocytochemical reaction for a specific blood vessel's marker per area of gingiva in recorded image (in pixels and $\mu \mathrm{m}^{2}$ ) for every patient and the entire group of patients, and was calculated also as a percentage $(\%)$.

Statistical analysis. At the first stage of statistical analysis, the compatibility of all the obtained results with Gauss's distribution was verified using the test of Shapiro-Wilk. The test of Levene demonstrated equality of variances for the three first parameters and, therefore, comparisons employed the $t$ test. Subsequently, parameters of descriptive statistics were calculated (arithmetic mean, standard deviation, median value, minimum and maximum values). Results of microscopic analysis data were compared between the studied subgroups (CP and $\mathrm{C} 1$ and/or C2) (independent samples) using the test of Mann-Whitney. In cases of dependent functions, the test of Wilcoxon was applied. Spearman's 
rank correlation was applied to correlate values of variables. The non-parametric test of Kruskal-Wallis was used in the $\mathrm{CP}$ group to compare the expression of individual markers as related to CAL value. A significant relationship or difference was accepted to reflect results at the significance level of $\mathrm{p} \leq 0.05$. The statistical analyses were carried out using Statistica PL v. 8.0 software.

\section{Results}

\section{Evaluation of inflammatory lesion intensity in gingival preparations obtained from patients with chronic periodontitis}

Inflammatory activity (grading) of three points was demonstrated in 39/61 (64\%) fragments of gingivae obtained from patients with CP; grading of two points characterized 18/61 (30\%) fragments; and grading of one point was noted in $4 / 61$ fragments $(6 \%)$. In the group of patients with $\mathrm{CP}$, no gingivae were found which were free of inflammatory infiltrates (and which would have been graded 0 ). In the gingivae of patients with $\mathrm{CP}$, the average intensity of inflammatory lesions in the semi-quantitative scale amounted to $2.57 \pm$ \pm 0.62 and it was significantly higher than grading in the gingivae of patients with a 'healthy' periodontium $(0.60 \pm 0.63)(\mathrm{p}=0.0001)$.

\section{Cellular localization of CD31 (PECAM-1), CD105 (endoglin) and VEGF in patients with chronic periodontitis}

Expression of CD31 was manifested both in cell membranes of ECs in HEVs (Figure 1A) and in ordinary gingival blood vessels (Figure 1B). Expression of PECAM-1 manifested the typical localization on lateral surfaces of HECs membranes, in the site of intercellular attachments (Figure 1A) and, which is noteworthy, on cell membranes of extravasally positioned leukocytes (mononuclear cells, individual neutrophils) of the inflammatory infiltrates in lamina propria (Figure 1A, arrow). Only a weak positive reaction was observed in the blood vessels of healthy gingival samples (Figure 1C).

Surface and cytoplasmic expression of CD105 (endoglin) in CP patients involved HEVs and ordinary gingival blood vessels (Figures 1D, E). Positive reaction was shown also in individual endothelial cells in blood vessels of healthy gingivae (Figure 1F).

In the $\mathrm{CP}$ group, expression of VEGF was best expressed in cells of inflammatory infiltrates (lymphocytes, macrophages) and not in ECs (Figure 1G, arrow). No VEGF expression was noted in epithelial cells of gingivae and/or periodontal pocket (Figure
1H). Only a very weak positive immunocytochemical reaction for the marker was observed in endothelial cells of $\mathrm{HEV}$ and/or typical blood vessels in gingivae with chronic periodontitis (data not shown).

In cases of gingival samples of group $\mathrm{C} 1$, expression of VEGF was negligible and pertained to individual cells of typical blood vessels of gingivae. In the C2 group, no positive reaction for VEGF was noted in HEVs and only a few lymphoid cells demonstrated a positive reaction.

\section{Cellular localization of ECs antigens in reactive lymph nodes (C2 group)}

Examination of vascular antigen expression in HEVs of reactive lymph nodes ( $\mathrm{C} 2$ group) permitted the presence of HEVs marker to be confirmed. The presence of CD31 (a variable reaction) was observed in vascular endothelium cells in every case of reactive lymph nodes (Figure 2A). The presence of endoglin was demonstrated in HECs and, sometimes, in cytoplasm of individual lymphocytes or monocytes/macrophages in organ parenchyma (Figure 2B).

\section{Comparison of vascular marker expression in $H E V$ s and in ordinary gingival ECs in CP patients}

No quantitative differences were detected in the expression of PECAM-1 in gingival HEVs, compared to the expression in ordinary blood vessels in patients with CP (Table 1).

A significantly elevated expression in $\mathrm{HEVs}$, compared to ordinary gingival ECs, involved endoglin. The entire VEGF expression (in ordinary vessels and inflammatory infiltration cells together) was significantly higher than expression in HEVs in the CP group (Table 1).

In gingival HEVs of the CP group, a significantly higher expression of PECAM-1 (4.58 \pm 3.72$)$ compared to those of endoglin $(1.64 \pm 1.61$; $\mathrm{p}=0.014)$ and VEGF $(0.87 \pm 2.70 ; \mathrm{p}=0.023)$, was demonstrated. The expression of endoglin in gingival HEVs was significantly higher than that of VEGF $(p=0.009)$.

\section{Comparison of vascular marker expression between CP group and negative control (C1)}

Gingivae of the $\mathrm{C} 1$ group contained only individual $\mathrm{HEV}$-type blood vessels in 2/15 patients. In CP patients, a significantly higher entire expression (HEVs and ordinary blood vessels) of PECAM-1, endoglin and VEGF was noted, compared to vessels of control gingivae $(\mathrm{p}=0.002, \mathrm{p}=0.001, \mathrm{p}=0.001$, respectively) (Table 1). 

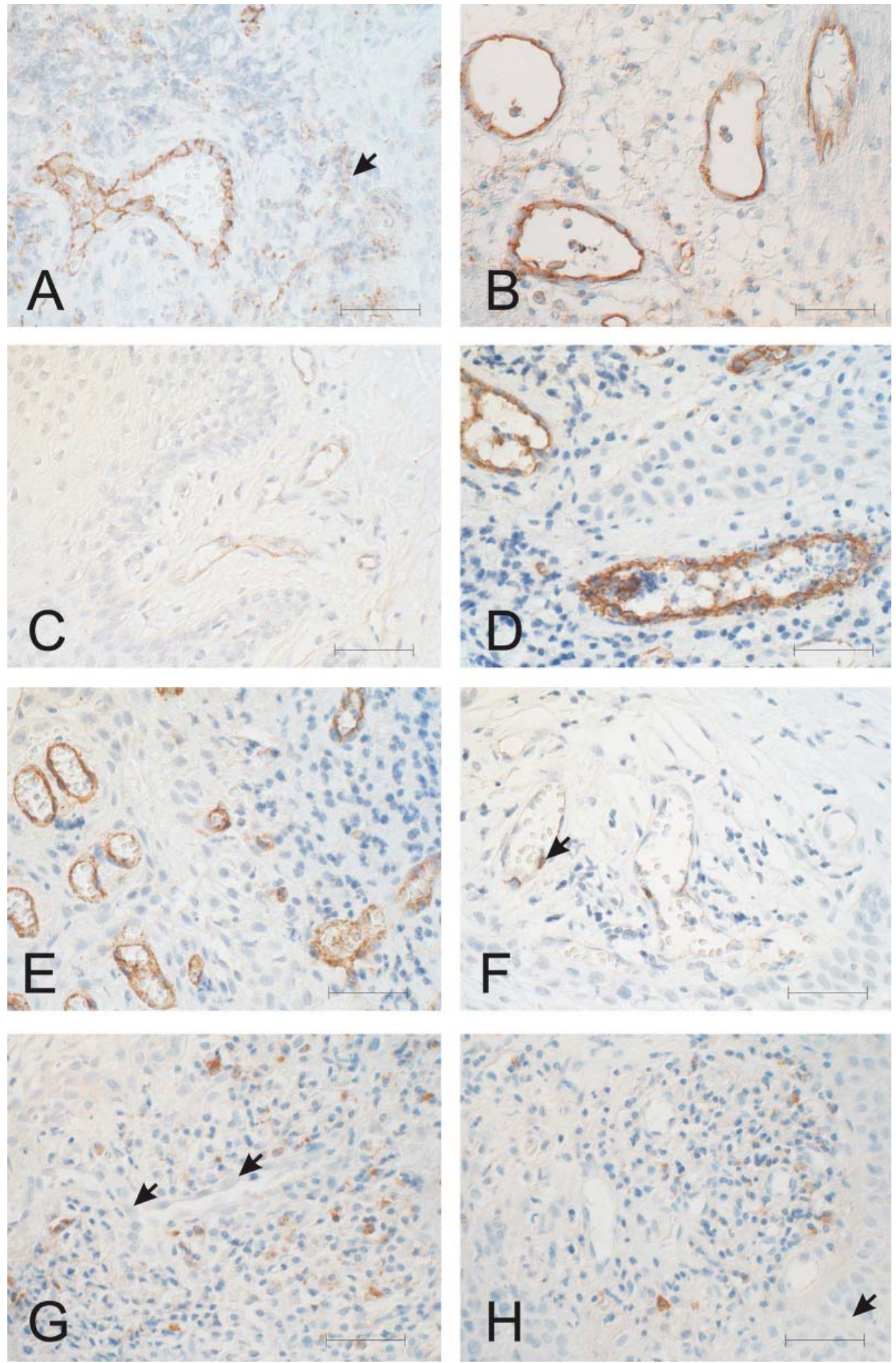

Figure 1. Cellular localization of HEV markers in fragments of gingivae in chronic periodontitis patients: (A) surface expression of PECAM-1 (CD31) in gingival postcapillary high endothelial venule (HEV) and in inflammatory cells (arrow); (B) CD31 expression in ordinary gingival blood vessels; (C) weak expression of CD31 in ordinary blood vessels in control group (C1); (D) membrane and cytoplasmic endoglin expression in HEVs; (E) endoglin localization in ordinary blood vessels; (F) weak positive expression of endoglin in ordinary blood vessels in fragment of healthy gingiva $(\mathrm{C} 1$ group); (G) VEGF expression mainly in inflammatory cells, but not in endothelial cells (arrow); (H) negative reaction for VEGF in epithelial cells of gingiva (arrow). ABC technique. Hematoxylin counterstained. Bar $=80 \mu \mathrm{m}$ 


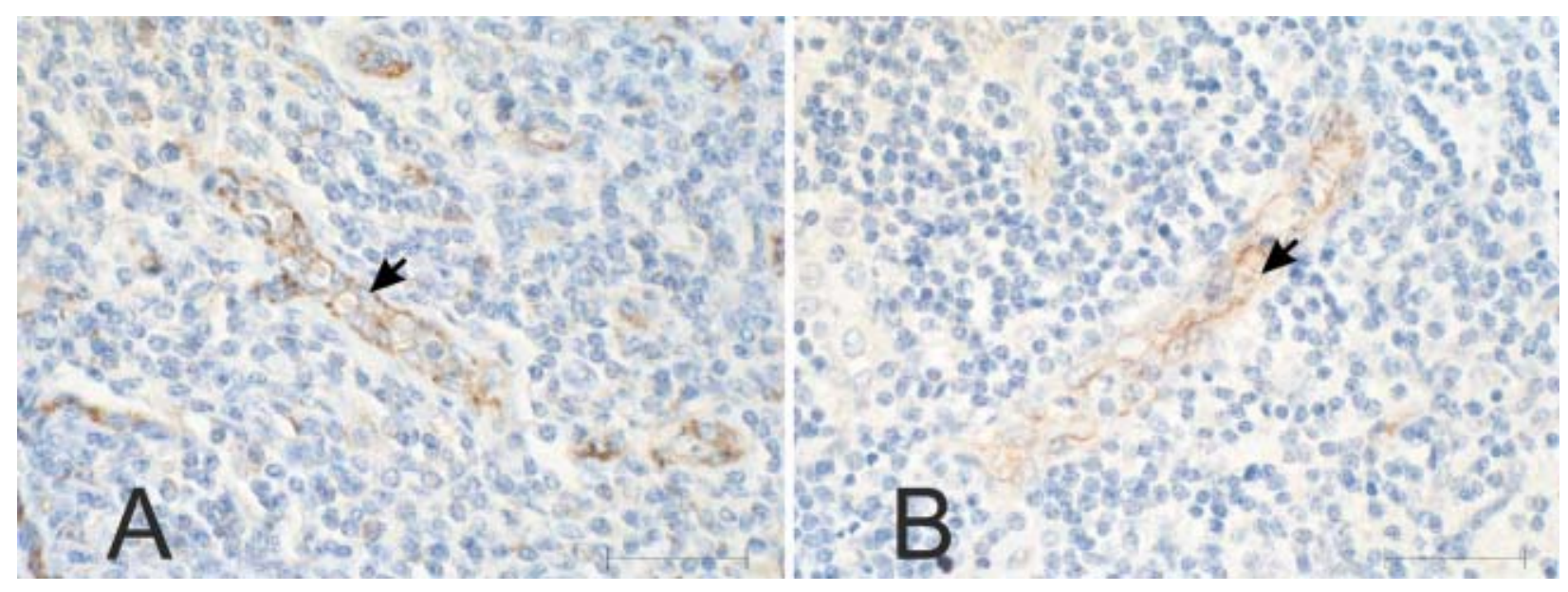

Figure 2. Cellular localization of vascular antigens in high endothelial postcapillary venules (HEVs) of reactive lymph nodes: (A) surface localization of PECAM-1 (CD31) (arrow); (B) surface localization of endoglin (arrow). ABC technique. Hematoxylin counterstained. Bar $=80 \mu \mathrm{m}$

Table 1. Comparison of vascular marker's expression (mean \% of immunocytochemical reaction \pm SD) in HEVs and ordinary gingival blood vessels in chronic periodontitis (CP group), and between gingival blood vessels in CP and in patients with healthy periodontia ( $\mathrm{C} 1$ group) and/or reactive lymph nodes (C2 group)

\begin{tabular}{|c|c|c|c|c|c|c|}
\hline Marker & Type of blood vessel & $\mathbf{C P}$ & C1 & $\mathbf{p}^{1}$ & C2 & $\mathbf{p}^{2}$ \\
\hline \multirow[t]{4}{*}{ PECAM-1 } & $\mathrm{HEVs}$ & $4.58 \pm 3.72$ & $1.33 \pm 1.88$ & $-*$ & $1.37 \pm 1.03$ & 0.010 \\
\hline & Ordinary & $3.94 \pm 3.31$ & $1.24 \pm 0.75$ & 0.019 & & \\
\hline & Significance level $p$ & 0.716 & $-*$ & & & \\
\hline & $\Sigma$ HEVs and ordinary & $8.34 \pm 6.58$ & $1.68 \pm 1.47$ & 0.002 & & \\
\hline \multirow[t]{4}{*}{ Endoglin } & HEVs & $1.64 \pm 1.61$ & $0.58 \pm 1.16$ & 0.039 & $1.60 \pm 1.83$ & 0.859 \\
\hline & Ordinary & $1.14 \pm 1.32$ & $0.42 \pm 0.87$ & 0.002 & & \\
\hline & Significance level $\mathrm{p}$ & 0.001 & $-*$ & & & \\
\hline & $\Sigma$ HEVs and ordinary & $2.78 \pm 2.61$ & $0.57 \pm 1.04$ & 0.001 & & \\
\hline \multirow[t]{4}{*}{ VEGF } & HEVs & $0.87 \pm 2.70$ & 0.00 & $-*$ & $0.01 \pm 0.01$ & 0.735 \\
\hline & Ordinary/inflammatory infiltrate cells & $3.18 \pm 4.53$ & $0.02 \pm 0.04$ & 0.001 & & \\
\hline & Significance level $\mathrm{p}$ & 0.022 & $-^{*}$ & & & \\
\hline & $\begin{array}{l}\Sigma \text { HEVs and ordinary/inflammatory } \\
\text { infiltrate cells }\end{array}$ & $3.57 \pm 5.02$ & $0.02 \pm 0.04$ & 0.001 & & \\
\hline
\end{tabular}

$\Sigma$ — sum; SD — standard deviation; *numerical power of one of the compared groups insufficient for statistical appraisal; $\mathrm{p}$ - significance level between HEVs and ordinary blood vessels expression of vascular markers inside CP and C1 groups; $\mathrm{p}^{1}$ — level of significance between CP and C1 groups; $\mathrm{p}^{2}$ - level of significance between CP and C2 groups (only HEVs)

\section{Comparison of vascular marker expression between $C P$ group and reactive lymph nodes $(C 2)$}

In CP patients, a significantly higher expression of PECAM-1 in HEVs was noted, compared to HEVs of $\mathrm{C} 2$ group. The remaining markers (endoglin and VEGF) manifested similar intensity of expression in the two compared groups (Table 1).

\section{Analysis of angiogenetic ratio (AR) (endoglin/PECAM-1 and VEGF/PECAM-1)}

A significantly higher ratio of the entire endoglin/ /PECAM-1 expressions (AR1) was manifested in the blood vessels in gingivae of $\mathrm{CP}$ patients compared to blood vessels of $\mathrm{C} 1$ group (Figure 3 ). On the other hand, no significant differences in AR were detected 


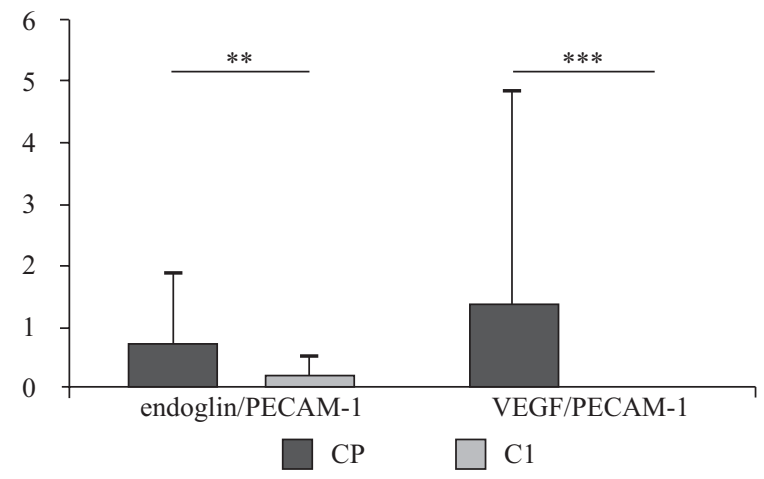

Figure 3. Ratios of entire endoglin/PECAM-1 expressions and of entire VEGF/PECAM-1 (angiogenetic ratio, AR1 and AR2) expressions in gingivae of patients with chronic periodontitis $(\mathrm{CP})$ and in patients with a healthy periodontium $(\mathrm{C} 1) ;{ }^{* *} \mathrm{p}=0.012 ; * * * \mathrm{p}=0.007$

between gingival HEVs and HEVs of reactive lymph nodes (C2) (Table 2).

Similarly, a significantly higher AR2, expressed by the ratio of entire VEGF/PECAM-1 expressions, was noted in the gingivae of patients with $\mathrm{CP}$ compared to blood vessels of the control group with healthy periodontia $(\mathrm{C} 1)$ (Table 2, Figure 3 ).

\section{Correlations between expression of vascular markers and inflammatory activity (grading) in gingivae of chronic periodontitis}

A weakly pronounced direct relationship was disclosed between inflammatory activity in gingiva (grading) and the expression of PECAM-1 (CD31) in ordinary blood vessels of gingiva (Spearman's rank correlation coefficient $r=0.389, p=0.049)$, but not expression of CD31 in HEV s of patients with CP $(r=0.383 ; p=0.059)$. A weak positive Spearman's correlation was noted also between the whole PECAM-1 expression and grading $(r=0.444, p=0.023)$.

Expression of the remaining studied vascular markers showed no correlation with intensity of inflammatory lesions in the gingivae of patients with $\mathrm{CP}$. Higher intensity of the entire cellular expression of all three markers was demonstrated in patients with more intense inflammatory lesions (grading 3), but the differences proved to be significant in Mann-Whitney's test only in the case of PECAM-1 (Table 3).

\section{Expression of vascular markers as related to clinical attachment level/loss (CAL) in patients with $\mathrm{CP}$}

Our study demonstrated no significant relationships between the total expression (in HEVs and in ordinary blood vessels) of vascular markers in the gingivae of patients with $\mathrm{CP}$ on the one hand and the values of CAL on the other. The values of total expression of vascular markers as related to CAL are presented in Table 4.

\section{Discussion}

In this study, localization of VEGF most frequently involved cells of inflammatory infiltrates (lymphocytes, macrophages), present in lamina propria of gingivae in patients with chronic periodontitis. This has corroborated the results of other authors who demonstrated VEGF in monocytes and macrophages [16]. It has not confirmed the results of those investigators who have observed prevalent cellular expression of VEGF in keratinocytes of epithelium in gingivae and/ /or periodontal pocket $[19,20]$. This study has noted only individual VEGF-immunopositive endothelial cells and they have represented almost exclusively HECs, and not ECs of ordinary gingival blood ves-

Table 2. Ratio of entire expression of endoglin to that of PECAM-1 (angiogenetic ratio, AR1) and of VEGF to that of PECAM-1 (angiogenetic ratio, AR2) in all groups of patients (CP, C1 and C2)

\begin{tabular}{|l|l|l|c|c|c|c|}
\hline Angiogenetic ratio & \multicolumn{2}{|c|}{ Group of patients/type of vessel } & Mean & Minimum & Maximum & SD \\
\hline \multirow{2}{*}{ Endoglin/PECAM-1 (AR1) } & $\mathrm{CP}$ & Whole vessels & $0.81^{*}$ & 0.02 & 4.40 & 0.98 \\
& & HEVs & $0.90^{* *}$ & 0.03 & 4.47 & 1.07 \\
\cline { 2 - 8 } & $\mathrm{C} 1$ & Whole vessels & 0.15 & 0.00 & 0.43 & 0.16 \\
& $\mathrm{C} 2$ & HEVs & 1.95 & 0.01 & 6.76 & 2.36 \\
\hline VEGF/PECAM-1 (AR2) & $\mathrm{CP}$ & & $0.42^{*}, * *$ & 0.00 & 16.47 & 3.50 \\
& $\mathrm{C} 1$ & Entire expression & 0.00 & 0.00 & 0.01 & 0.00 \\
& $\mathrm{C} 2$ & & 0.01 & 0.00 & 0.04 & 0.02 \\
\hline
\end{tabular}

$\mathrm{SD}$ - standard deviation; In case of AR1 * $\mathrm{p}=0.012$ between $\mathrm{CP}$ and $\mathrm{C} 1$ group; $* \mathrm{p}=0.324$ between $\mathrm{CP}$ and $\mathrm{C} 2$ group (exclusively in $\mathrm{HEV}$ ); In case of $\mathrm{AR} 2 * \mathrm{p}=0.007$ between $\mathrm{CP}$ and $\mathrm{C} 1 ; * \mathrm{p}=0.006$ between $\mathrm{CP}$ and $\mathrm{C} 2$ 
Table 3. Comparison of entire expression (in HEVs and in ordinary gingival blood vessels) of vascular markers as related to inflammation activity (grading) in patients with chronic periodontitis (CP)

\begin{tabular}{|l|c|c|c|c|c|c|c|}
\hline Marker & Grading & $\mathbf{n}$ & Mean & Minimum & Maximum & SD & $\mathbf{p}$ \\
\hline PECAM-1 & 1 & 0 & - & - & - & - & $-^{*}$ \\
& 2 & 8 & 5.04 & 0.58 & 18.10 & 5.90 & 0.026 \\
& 3 & 18 & 9.80 & 1.01 & 26.63 & 6.48 & \\
\hline Endoglin & 1 & 4 & 1.14 & 0.00 & 1.80 & 0.83 & $-{ }^{*}$ \\
& 2 & 18 & 2.46 & 0.00 & 8.56 & 2.33 & 0.592 \\
& 3 & 39 & 3.10 & 0.00 & 9.45 & 2.80 & \\
\hline VEGF & 1 & 4 & 3.07 & 0.00 & 6.95 & 3.17 & $-^{*}$ \\
& 2 & 12 & 2.46 & 0.13 & 10.30 & 2.86 & 0.769 \\
& 3 & 26 & 4.16 & 0.00 & 23.81 & 5.96 & \\
\hline
\end{tabular}

The table includes: number of studied gingival preparations with positive reaction (n), values of mean, minimum and maximum marker expression expressed in \% of positive immunocytochemical reaction area, standard deviation (SD) and significance level (p); Grading was evaluated in a 4-point semi-quantitative scale (see Material and methods); *numerical force in one of compared group was insufficient for statistical analysis

Table 4. Entire expression of vascular markers (in HEVs and in ordinary blood vessels) as related to clinical attachment level/loss (CAL) value

\begin{tabular}{|l|c|c|c|c|c|c|c|}
\hline Marker & CAL & $\mathbf{n}$ & Mean & Minimum & Maximum & SD & p \\
\hline PECAM-1 & 1 & 2 & 2.44 & 2.26 & 2.62 & 0.25 & \\
& 2 & 3 & 5.81 & 0.58 & 8.98 & 4.57 & $-^{*}$ \\
& 3 & 21 & 9.26 & 1.01 & 26.63 & 6.84 & \\
\hline Endoglin & 1 & 6 & 1.89 & 0.39 & 4.67 & 1.62 & \\
& 2 & 10 & 1.72 & 0.00 & 4.97 & 1.89 & 0.204 \\
& 3 & 45 & 3.14 & 0.00 & 2.45 & 2.79 & 1.18 \\
\hline VEGF & 1 & 4 & 1.21 & 0.00 & 6.95 & 2.65 & 0.575 \\
& 2 & 6 & 2.44 & 0.57 & 23.81 & 5.55 & \\
\hline
\end{tabular}

The table includes: number of studied gingival preparations with positive reaction (n), values of mean, minimum and maximum marker expression expressed in \% of positive immunocytochemical reaction area, standard deviation (SD) and significance level (p); CAL 1: $\leq 3 \mathrm{~mm}$, CAL 2: 4-6 mm, CAL 3: $\geq 7 \mathrm{~mm}$; *numerical force in one of compared group was insufficient for statistical analysis

sels. In gingival samples obtained from patients with a healthy periodontium, the expression of VEGF was negligible and involved individual flat ECs of gingival blood vessels, in accordance with the results of other investigators [16, 21]. However, the obtained results do not allow the confirmation of localization of VEGF mainly in microvessel endothelium or localization manifesting higher intensity than that in blood vessels of control gingivae, as demonstrated by other authors [21]. The study by Artese et al. provided no source of the antibodies applied to localize VEGF in the studied tissue material, which may be of significance for localization of a specific isoform of the protein. In our study, we applied monoclonal mouse anti-human antibodies for parallel detection of three VEGF isoforms: VEGF-A (VEGF-121), VEGF-B (VEGF-165) and VEGF-C (VEGF-189) produced by DAKO (clone VG1). Also the applied antibody dilution was 1:50 in the work of Artese et al., while we applied a dilution of 1:100 [21]. Similarly to our results, the authors failed to note epithelial localization of VEGF, as documented and highlighted in the study by Lucerini et al. [20]. The latter study was based on the use of frozen sections (and not paraffin sections, as in our study) and VEGF-specific antibodies produced by Santa Cruz Biotechnology. The latter authors demonstrated VEGF localization mainly in cells of gingival epithelium, while endothelial cells showed moderate positivity [20].

In chronic inflammatory periodontal diseases, the expression of VEGF began to be investigated in the late 1990s [33]. Using immunocytochemical techniques, the presence of VEGF was demonstrated at the time in a wide range of cells, beginning at endot- 
helium, through neutrophils and plasma cells, to cells of gingival epithelium and/or epithelial cells of the periodontal pocket [33].

In our opinion, it is important to confirm the augmented total expression of the vascular marker in periodontitis compared to gingivae with a healthy periodontium, the conclusion which seems to stem from all quoted results of studies, including our own. We cannot refer to results related to gingivae in diabetic patients, suffering in addition from periodontitis, since we had no access to such tissue material. Gingivae of such patients have been found to contain even more numerous VEGF-immunopositive cells compared to 'pure' CP and the control [20].

In our study, we must emphasize the novelty of the applied spatial visualization technique for reproducible and reliable detection of intensity manifested of vascular marker expression in gingival mucosa [32]. The hard disc-archived histological preparations can also be analyzed from other points of view, e.g. by measuring the HEV-type blood vessel number, the number of 'standard' blood vessels and/or their diameter, and the presence of inflammatory infiltrates, due to the presence of positive immunocytochemical reaction.

The results of some authors have documented increased concentrations of VEGF in gingivitis, which manifested correlation with depth of periodontal pocket [14]. This was associated with increased numbers of blood vessels and higher VEGF/IL-6 ratio in gingivae of patients with 4-6 mm depth of periodontal pocket, compared to healthy gingival fragments [14]. Similar correlations between concentration of VEGF in gingival fluid in patients with $\mathrm{CP}$ and clinical attachment level/loss (CAL) have been noted by other authors [15].

The results of this study have failed to document significant correlations between the expression of VEGF and intensity of inflammatory lesions (grading) in patients with CP or CAL value even if a tendency could have been noted for higher expression of VEGF in most pronounced inflammatory lesions (grading 3 ) or in the most pronounced clinical attachment level/loss (CAL3) in patients with CP. The absence of statistical significance in the changes might merely reflect the prevalence of gingival samples with the most pronounced values of both grading (64\% grading 3) and CAL (74\% - CAL3), compared to individual gingival samples with less pronounced destruction of periodontal tissues.

The results would require confirmation on a more extensive tissue material or using other models of studies.

In ECs of HEV in reactive lymph nodes collected in this study, no positive reaction for VEGF was not- ed and only a few lymphoid cells manifested the positive reaction. Thus, the observations of other authors were only partly confirmed [19].

Our results have demonstrated a higher expression of VEGF in patients with CP, compared to control groups, which might confirm the potential involvement of local VEGF production in the remodeling of gingival blood vessels, possibly including generation of HEV-type blood vessels. On the other hand, we are unable to agree with the observations of other authors that local production of VEGF is less pronounced in more advanced inflammatory lesions in the periodontium $[19,33]$.

Similarly to results of other authors [34], the expression of PECAM-1 (CD31) in this study has been particularly pronounced on the surface of venous ECs (including HEVs) and in inflammatory cells. Indirectly, the results of this study may be related to analysis of gene expression profiles typical for HECs, as described in the literature [29]. In the quoted publication, a partial similarity was shown between gene expressions typical for HEC and for CD31-positive cells of flat endothelium. The common gene profile of the two types of endothelium (i.e. the high and the flat) included the following markers: endoglin, CD32, CD63, L6 antigen and ICAM-1 [29]. Similarly, in our study we have demonstrated that endoglin (CD105) represents a common marker of HEC endothelial cells and flat endothelium $\left(\mathrm{CD} 31^{+}\right)$in ordinary blood vessels in gingivae of patients with chronic pathology of the periodontium. Nevertheless, our results, in contrast to those of Izawa et al. [29], have shown a significantly lower mean expression of endoglin in gingival HEVs compared to the expression of CD31 in the same gingival blood vessels. In reactive lymph nodes (group C2), the intensity of endoglin expression has not differed significantly from the expression of other HEVs markers.

It remains difficult to relate the results of this study to literature data, which have described expression of endoglin in ECs present in pulpar blood vessels of patients with CP and not in gingival blood vessels [27]. Similarly to the gingivae of our patients, the authors demonstrated endoglin expression of a variable intensity which manifested no significant correlation with expression of other adhesion molecules or intensity of inflammatory lesions [27].

Assuming the potential involvement of CD105 (endoglin) and CD31 (PECAM-1) in neoangiogenesis, and attempting to more accurately define the role of endothelial expression of the molecules in patients with CP, following the study of Tachezy et al. (2010) [35], CD105/CD31 angiogenetic ratio (AR1) was established to determine the proliferation fraction of 
the endothelium. A significantly higher AR1 has characterized gingival blood vessels of patients with CP, compared to the $\mathrm{C} 1$ group. On the other hand, no significant difference has been noted in AR1 calculated exclusively for HEVs of gingivae with $\mathrm{CP}$ and HEVs of reactive lymph nodes (group C2). Also, no differences in the angiogenetic ratio have been disclosed between the $\mathrm{CP}$ group and patients with a healthy periodontium taking into account CD105/ /CD31 ratio in ordinary blood vessels in both examined groups. Thus, differences in the total AR1 between patients with $\mathrm{CP}$ and the control group with a healthy periodontium seem to be related to the presence of HEVs in the inflamed gingivae and, as a consequence, to the increased expression of HEV markers.

We decided also to examine the index of VEGF/ /CD31 (AR2), considering the documented inductive effect of VEGF on angiogenesis in vivo and involvement of the growth factor in the development (in specific conditions) of venous blood vessels [36]. The AR2, defined in this manner, has been found to manifest even greater differences compared to those noted in AR1, in the group of patients with chronic periodontitis both as compared to $\mathrm{C} 1$ or $\mathrm{C} 2$ control group. A surprisingly low expression of VEGF has been demonstrated in reactive lymph nodes, which has also resulted in a low value of the entire VEGF/CD31 index. Indirectly, the results point to a significant role of examining the angiogenetic ratio in demonstration of an increased angiogenesis in chronic inflammatory conditions, in which new blood vessels appear, including venules with a high endothelium.

The increased (as compared to the negative control (C1)) angiogenetic index (endoglin/PECAM-1 and VEGF/PECAM-1) in the gingivae of patients with chronic periodontitis provides evidence for a stimulated angiogenesis in inflammatory altered periodontal tissues.

The comparable value of AR1 (CD105/CD31) in HEV type blood vessels in various organs (gingiva, lymph nodes), may confirm the similarity of functions of both studied molecules in angiogenesis and leukocyte migration through HEVs during inflammation. Summing up, the role of examined vascular markers is related, above all, to the stimulation of angiogenesis (including the proliferation of HEVs, which play an important additional function in transendothelial migration of leukocytes) in the course and progression of periodontitis. The introduction of both indices of vascular proliferation (endoglin/CD31 and VEGF/CD31) has permitted the confirmation of the finding of stimulated vascular proliferation in this human disease.

\section{References}

1. Page RC, Beck JD. Risk assessment for periodontal diseases. Int Dent J. 1997;47:61-87.

2. Slots J, Kamma JJ, Sugar C. The herpesvirus-Porphyromonas gingivalis-periodontitis axis. J Periodontal Res. 2003;38:318-323.

3. Silva N, Dutzan N, Hernandez M et al. Characterization of progressive periodontal lesions in chronic periodontitis patients: levels of chemokines, cytokines, matrix metalloproteinase-13, periodontal pathogens and inflammatory cells. J Clin Periodontol. 2008;35:206-214.

4. Zoellner HF, Chapple CC, Hunter N. Microvasculature in gingivitis and chronic periodontitis: disruption of vascular networks with protracted inflammation. Microsc Res Tech. 2002;56:15-31.

5. Zoellner HF, Hunter N. Vascular expansion in chronic periodontitis. J Oral Pathol Med. 1991;20:433-437.

6. Demmer RT, Desvarieux M. Periodontal infections and cardiovascular disease: the heart of the matter. J Am Dent Assoc. 2006;137(Suppl. 1):14-20.

7. Bahekar AA, Singh S, Saha S, Molnar J, Arora R. The prevalence and incidence of coronary heart disease is significantly increased in periodontitis: a meta-analysis. Am Heart J. 2007; 154:830-837.

8. D'Aiuto F, Graziani F, Tete' S, Gabriele M, Tonetti MS. Periodontitis: from local infection to systemic diseases. Int J Immunopathol Pharmacol. 2005;18:1-12.

9. Kasprzak A, Surdacka A, Tomczak M et al. Analysis of marker expression in high endothelial venules in chronic periodontitis. Adv Clin Exp Med. 2011;20:275-284.

10. Wynne SE, Walsh LJ, Seymour GJ. Specialized post-capillary venules in human gingival tissue. J Periodontol. 1988;59:328-331.

11. Polverini PJ. The pathophysiology of angiogenesis. Crit Rev Oral Biol Med. 1995;6:230-247.

12. Ferrara N. Vascular endothelial growth factor and the regulation of angiogenesis. Recent Prog Horm Res. 2000;55:15-35.

13. Ferrara N, Gerber HP, LeCouter J. The biology of VEGF and its receptor (review). Nat Med. 2003;9:669-676.

14. Johnson RB, Serio FG, Dai X. Vascular endothelial growth factors and progression of periodontal diseases. J Periodontol. 1999;70:848-852.

15. Prapulla DV, Sujatha PB, Pradeep AR. Gingival crevicular fluid VEGF levels in periodontal health and disease. J Periodontol. 2007;78:1783-1787.

16. Unlü F, Güneri PG, Hekimgil M, Yesilbek B, Boyacioğlu H. Expression of vascular endothelial growth factor in human periodontal tissues: comparison of healthy and diabetic patients. J Periodontol. 2003;74:181-187.

17. Suthin K, Matsushita K, Machigashita M et al. Enhanced expression of vascular endothelial growth factor by periodontal pathogens in gingival fibroblasts. J Periodontal Res. 2003;38:90-96.

18. Booth V, Young S, Cruchley A, Taichman NS, Paleolog E. Vascular endothelial growth factor in human periodontal disease. J Periodontal Res. 1998;33:491-499.

19. Chapple CC, Kumar R, Hunter N. Vascular remodelling in chronic inflammatory periodontal disease.J Oral Pathol Med. 2000;29:500-506.

20. Lucarini G, Zizzi A, Aspriello SD et al. Involvement of vascular endothelial growth factor, CD44 and CD133 in periodontal disease and diabetes: an immunohistochemical study. J Clin Periodontol. 2009;36:3-10. 
21. Artese L, Piattelli A, de Gouveia Cardoso LA et al. Immunoexpression of angiogenesis, nitric oxide synthase, and proliferation markers in gingival samples of patients with aggressive and chronic periodontitis. J Periodontol. 2010; 81:718-726.

22. Nagashima M, Yoshiro S, Ishiwata T, Asano G. Role of vascular endothelial growth factor in angiogenesis of rheumatoid arthritis. J Rheumatol. 1995;22:1624-1630.

23. Yun P, Decarlo A, Chapple C, Hunter N. Functional implication of the hydrolysis of Platelet Endothelial Cell Adhesion Molecule 1 (CD31) by gingipains of Porphyromonas gingivalis for the pathology of periodontal disease. Inf Immun. 2005;73:1386-1398.

24. Newman PJ, Newman DK. Signal transduction pathways mediated by PECAM-1: New roles for an old molecule in platelet and vascular cell biology. Arterioscler Thromb Vasc Biol. 2003;23:953-964.

25. Paprocka M, Duś D. Phenotypic and functional features of endothelial cells (Article in Polish). Post Biol Kom. 2000;27:247-260.

26. Gemmell E, Walsh LJ, Savage NW, Seymour GJ. Adhesion molecule expression in chronic inflammatory periodontal disease tissue. J Periodontal Res. 1994;29:46-53.

27. Tasman F, Dagdeviren A, Kendir B, Ozçelik B, Ataç A, Er N Endothelial cell adhesion molecules in human dental pulp: a comparative immunohistochemical study on chronic periodontitis. J Endod. 1999;25:664-667.

28. Bernabeu C, Conley BA, Vary CP. Novel biochemical pathways of endoglin in vascular cell physiology. J Cell Biochem. 2007;102:1375-1388.
29. Izawa D, Tanaka $\mathrm{T}$, Saito $\mathrm{K}$ et al. Expression profile of active genes in mouse lymph node high endothelial cells. Int Immunol. 1999;11:1989-1997.

30. Hsu SM, Raine L, Fanger H. Use of avidin-biotin-peroxidase complex $(\mathrm{ABC})$ in immunoperoxidase techniques: a comparison between $\mathrm{ABC}$ and unlabeled antibody (PAP) procedures. J Histochem Cytochem. 1981;29:577-580.

31. Kasprzak A, Małkowska A, Hausmann M, Przybyszewska W, Zabel M, Stopa J. Detection of cell phenotypes and pro-inflammatory cytokines (IL- $1 \alpha$, TNF- $\alpha$, IL-6) in chronic periodontitis using ImmunoMax technique. Pol J Environ Stud. 2007; 16:328-336.

32. Kaczmarek E, Strzelczyk R. From two to three-dimensional visualisation of structures in light and confocal microscopy - applications for biomedical studies. In: Mendez-Vilas A, LabajosBroncano L, eds. Current issues on multidisciplinary microscopy research and education. FORMATEX microscopy book series no. II Formatex Research Centre; Badajoz 2005:289-295.

33. Bonakdar MP, Barber PM, Newman HN. The vasculature in chronic adult periodontitis: a qualitative and quantitative study. J Periodontol. 1997;68:50-58.

34. Tanaka Y, Albelda SM, Horgan KJ et al. CD31 expressed on distinctive $\mathrm{T}$ cell subsets is a preferential amplifier of beta 1 integrin-mediated adhesion. J Exp Med. 1992;176:245-253.

35. Tachezy M, Reichelt U, Melenberg T, Gebauer F, Izbicki JR, Kaifi JT. Angiogenesis index CD105 (Endoglin)/CD31 (PECAM-1) as a predictive factor for invasion and proliferation in intraductal papillary mucinous neoplasm (IPMN) of the pancreas. Histol Histopathol. 2010;25:1239-1246.

36. Kume T. Specification of arterial, venous, and lymphatic endothelial cells during embryonic development. Histol Histapathol. 2010;25:637-646.

Submitted: 29 December, 2011 Accepted after reviews: 27 April, 2012 